several authors reporting possible correlations among macrophagic infiltration, neovascularity and prognosis.

The aims of this study are the analysis of neoangiogenic pattern of indolent NHL patients (pts) and the role of stromal cells in neovascularization at diagnosis and relapse/progression disease, in order to identify a subset of pts that could benefit by integrated anti-angiogenic treatment.

Methods: Nodal and bone marrow biopsies from 6 follicular (FL) and 7 small lymphocytic (SLL) NHL's pts were selected and studied at diagnosis and disease relapse/progression ( 9 males and 5 females; mean age at diagnosis 59.8 yrs; $13 / 13$ stage III/IV; low/intermediate FLIPI $7 / 13$ pts, high risk FLIPI $6 / 13$ pts, 8 relapsed pts with median PFS 33.37 months and 5 refractories).

We evaluated both neoangiogenesis by immunohistochemistry (anti-CD34, vascular hot spots/field), confirmed by morphometric analysis, and monocytic-macrophagic infiltrate (anti-CD68).

Results: At diagnosis we observed an angiogenic activity in all nodal samples with an homogeneous vascular distribution in SLL and perifollicular in FL (mean of 27.50 vascular hot spots (vhs)/field; median of $20 \mathrm{vhs} /$ field in nodal biopsies).

Moreover, at onset patients with low/intermediate prognostic risk showed a higher vhs/field. The number of vhs/field increased in all cases at relapse/progressive disease (mean 39,6; median 44 vhs/field) with statistical significance (T-test $p=0.0049$; Wilcoxon signed-rank test $p=0.03$ ) (Fig 1 ). On the contrary, no significant angiogenic activity was reported in bone marrow neither at diagnosis nor at relapse/progression. The distribution of monocyticmacrophagic infiltrate was heterogeneous in nodal samples with a focal or diffuse pattern and with a different match with the neovessels The percentage of monocytic-macrophagic infiltrate showed a poor increment at relapse/progression and seemed to lack any correlation with clinical features.

Conclusions: These preliminary data could justify the employment of angiogenesis analysis in the prognostic stratification of indolent NHL and hypothesize the use of anti-angiogenic drugs in patients relapsed or refractory to treatment. Larger series are warranted to confirm these data.

DIAGNOSIS

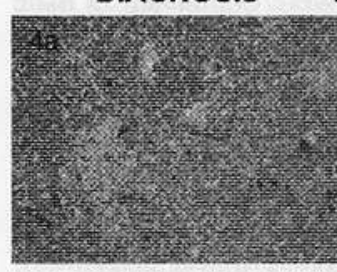

RELAPSE/PROGRESSION
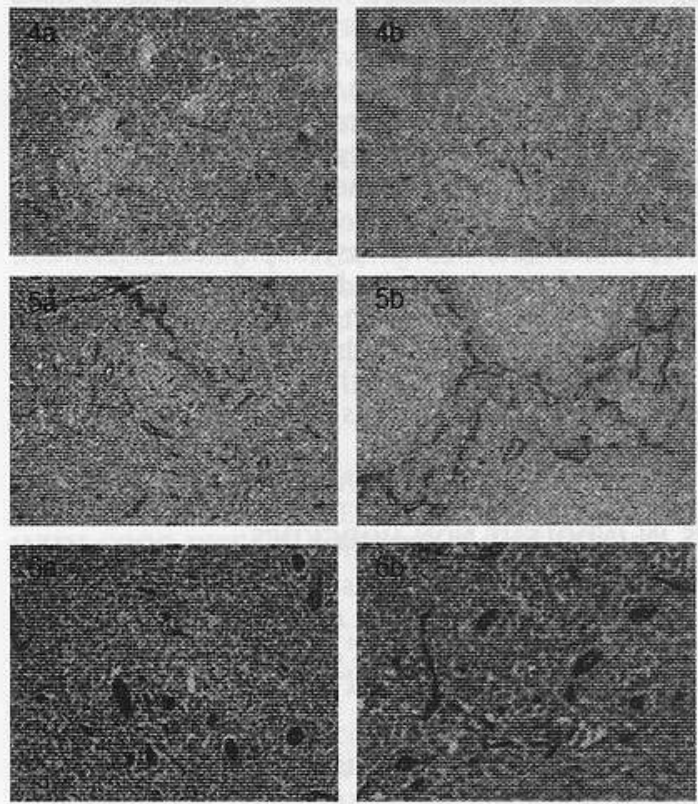

\begin{tabular}{llll}
\hline CASE & DIAGMOSIS & RELAPSE/PROGR. & DFS mts \\
\hline 4 & 20 & 50 & 13 \\
5 & 50 & 50 & 28 \\
6 & 20 & 38 & 28 \\
\hline
\end{tabular}

78

FIRST-LINE MODIFIED SCHEDULE OF GEMCITABINE INDUCES HIGH CLINICAL BENEFIT RATE WITHOUT SIGNIFICANT TOXICITY IN ELDERLY OR NOT ELDERLY ECOG PS 2 PATIENTS WITH ADVANCED NON-SMALL CELL LUNG CANCER

A. Nacci ${ }^{1}$, E. Mazzoni ${ }^{1}$, P. Rizzo ${ }^{1}$, L. Orlando ${ }^{1}$, F. Sponziello ${ }^{1}$, N. Calvani ${ }^{1}$, P. Schiavone ${ }^{1}$, M. D'Amico ${ }^{1}$, A. Marino ${ }^{1}$, P. Fedele ${ }^{1}$, M.C. Chetri ${ }^{1}$, M. Cinefra ${ }^{1}$, S. Cinieri ${ }^{1} .{ }^{1}$ U.O.C. Oncologia Ospedale "Senatore Antonio Perrino", Brindisi, Italy

Background: Monochemotherapy with gemcitabine (Gem) is often the treatment of choice in elderly or poor performance status (PS) patients with advanced non-small cell lung cancer (NSCLC). Our study was aimed to assess the efficacy and tolerability of a modified schedule of Gem using a lower dose than standard.

Methods: From May 2009 through April 2010, thirty-one patients ( 25 males and 6 females with a median age of 75 years ranging from 64 to 84 ) with advanced NSCLC (stage IIIB $38.8 \%$ and IV 61.2\%) were enrolled. Histology was: squamous $38.8 \%$, adenocarcinoma $29 \%$, large cell $6 \%$, undifferentiated $3 \%$, undetermined $24.8 \%$. Only six patients $(19.3 \%)$ had a WHO PS 0 whereas fourteen $(45.2 \%)$ were PS 1 and eleven (35.5\%) PS 2. All patients received first-line chemotherapy with 6 cycles of Gem $1000 \mathrm{mg} / \mathrm{sq}$ on days 1 and 8 every 4 weeks.

Results: At the time of analysis 26 patients were evaluable for response. Partial response (PR) was achieved in 4 patients (15.4\%), stable disease $>12$ weeks (SD) in $11(42.3 \%)$ whereas 11 had progressive disease (42.3\%). Importantly, the clinical benefit rate (PR+SD) was $57.7 \%$. Tumour markers (CEA and NSE) were high in 21 patients with a reduction in their values observed in 8 of them (38\%). Both pain and PS improved in 4 patients (15.4\%) whereas $16(53 \%)$ had an improvement in pain with no worsening of PS. We observed only grade 2 WHO haematological toxicities including anemia, leucopenia, neutropenia and trombocytopenia. Not-neutropenic fever occurred in 4 patients (13.3\%). Overall, we did not observe any not-haematological treatment-related event.

Conclusions: Our data show that a modified schedule of Gem with a lower dose intensity than standard may be beneficial in terms of both disease control and tolerability when employed in elderly or PS 2 patients with advanced NSCLC.

79

\section{VUS VARIANTS IN BRCA GENES OF HEREDITARY BREAST/OVARIAN CANCER}

M. Perez ${ }^{1}$, N. Margarese ${ }^{1}$, V. Calò ${ }^{1}$, L. Bruno ${ }^{1}$, L. La Paglia ${ }^{1}$, S. Cimino ${ }^{1}$, L.R. Corsini ${ }^{1}$, M. Terrasi ${ }^{1}$, D. Fanale ${ }^{1}$, V. Amodeo ${ }^{1}$, L. Insalaco ${ }^{1}$, L. Napoli' ${ }^{1}$, F. Di Gaudio' ${ }^{1}$, F. Di Piazza' ${ }^{1}$, M.C. Miraglia', V. Bazan', A. Russo'. 'Regional Reference Centre for the Biomolecular Characterization and Genetic Screening of Hereditary Tumors, University of Palermo, Palermo, Italy

Background: Germline mutations in BRCA1/2 genes are responsible for a large proportion of hereditary breast and/or ovarian cancers (HBOC syndrome). Many highly penetrant predisposition alleles have been identified and include frameshift or nonsense mutations which lead to the translation of a truncated protein. Other alleles contain missense mutations which result in amino acid substitution and intronic variant with splicing effect. The finding of variant of uncertain/unclassified significance (VUS) is a possible result that can complicate rather than improve the risk assessment process. VUSs are mainly missense mutations but also include a number of intronic variants and in-frame deletions and insertions.

Methods: A total of 141 unrelated families affected with breast and/or ovarian cancer proved to be eligible for a inclusion in an ongoing study and were screened for germline mutations in BRCA1 and BRCA2 at the "Regional Reference Center for the Characterization and Genetic Screening of Hereditary Tumors" at the University of Palermo using automatic direct sequencing. Moreover we collected a control population consisting of 50 index cases without a familial history of cancer and we analyzed this control group for the presence of VUS.

Results: We analyzed $B R C A 1$ and $B R C A 2$ genes and we found five VUS in the BRCA1 gene (A521T, Y179C, N550H, V740L, A622V) and twelve in BRCA2 gene (A22T, Y42C, A 2466V, 3010I, T200I, IVS24-16T>C, R2034C, IVS25-12T>G, IVS 2-7T>A, P2639A, IVS 
11-19delAT, IVS24-16T>C), classified in BIC database. Fifty healthy individuals were analyzed but any VUS didn't find, suggesting no association of these alteration with HBOC syndrome.

Conclusion: We indentified seventeen VUS in BRCA1/BRCA2 genes that could development of HBOC syndrome. The study of these variants in fifty healthy individuals confirms this hypothesis. VUS category posed many problems in genetic counseling due to the difficulty in the interpretation of the new or rare variants of BRCA genes. Evidence from functional assays other study are necessary to classify VUS into deleterious/high or neutral/low clinical significance. 